\title{
Place, Media and Activity
}

\section{Position Paper for the ECSCW 2001 'Work/Place' Workshop}

\author{
Matthew Chalmers \\ Computing Science, University of Glasgow, U.K. \\ http://www.dcs.gla.ac.uk/ matthew
}

\begin{abstract}
The workshop's call for participation points out the changes in work and society related to the development of new technologies, forms of work and patterns of activity. The traditional notion of workplace is challenged by the convergence and intercommunication of technological devices, as are our approaches to design and theory. I'd like to argue that this is only problematic because the traditional view is rather reductionist and simplistic. I'd like explore a different notion of what place is, and hence workplace. This builds on and extends the discussion of space and place of Harrison and Dourish, taking an approach based on post-structuralist semiology that takes fuller account of the interdependence of media in human activity than older HCI and CSCW. People's activity continually combines and cuts across different media, interweaving those media and building up the patterns of association and use that make meaning. How people act and work is determined by the full combination of media that they can use, and hence a narrow focus on space as the paramount determinant of activity underrates the influence of other media. 'Place' is not solely determined by the physical medium of space. A person's work or activity may be influenced by the configuration of space around them and the interactions that space affords, but also by books, telephones, hypermedia, 3D computer graphics and so forth. Recent technological developments heighten or highlight a phenomenon already familiar through analysis of the effect of older media such as written text, maps and cinema. This stance is now being explored in the City project, which focuses on a treatment of the city that deliberately blurs the boundaries between physical and digital spaces. By combining mobile computers, hypermedia and virtual environments in one system, and allowing each person using each medium or combination of media to interact with people using every other medium, our system is both driven by our theoretical approach and driving the development of theory.
\end{abstract}

\section{Space and Place}

A city's meaning is not just in its bricks and mortar, but also in our understanding and use of the information about it. Physical space is just one of the media that has affordances, and at any time one is likely to have symbols in a number of media available for interpretation and use. As I step out of a train station into a city square, the printed map in my hand, the voice of a colleague on my phone, and the signs informing me of exit routes and exciting shopping opportunities are all open for my interpretation and action. Temporally, symbols in an even broader range of media may influence me, as my interpretation and activity at any point in time is influenced by my past experience and my expectations of the future. Past experience may include my previous visits to that city, seeing television footage of the city, experience of magazines, books and films about urban life, and so forth. My language and culture, spanning all media, affects me as much as the immediate perception of physical phenomena such as spatial form, sound and light. For almost a century, a fundamental tenet of linguistics and semiology has been that such low-level physical phenomena are interpreted by a person via language, by the patterns of activity that the person has experienced in any and all media.

We can now take a semiological view of the distinction between space and place (Harrison \& Dourish 1996). We present a small and modified part of a forthcoming JCSCW paper (Chalmers 2001). Harrison and Dourish suggested that the significance and utility of a space increases when the people people build up a past that involves it, a history of experiences that allows the space to obtain the richer quality of 'place.' This change involves supporting the development of 'appropriate behavioural framing,' the emergent 
patterns of human behaviour and interaction that offer understandings of the space. It is important to note that their discussion refers not only to physical environments but also media spaces, information spaces such as Usenet groups, and hybrids of the physical and the virtual. In developing technical mechanisms to support awareness, achieving this quality of 'place' is an important goal.

They focus on information spaces that employ aspects of physical spaces in order to support cooperative work, and suggest that naïve mimicry of the physical world does not significantly help in making a useful place out of a sterile information space. One critical factor, they say, is "support for adaptation and appropriation of the technology by user communities." This leads to the development of a "communallyheld sense of appropriate behaviour, and a context for engaging in and interpreting action."

Harrison and Dourish go on to build up a complex set of terms and concepts, such as 'spaceless places,' in interpreting issues of technology design and information representation in terms of space and place. We take a slightly different approach to understanding the development and support of awareness. We treat a space or place as a medium for significant action, and as an addition to the other media that are interwoven in our activity and language i.e. we consider spatial structure and spatial action as parts of language. We consider 'space' to be one of the physical phenomena which has the potential to be used symbolically, and so consider a 'place' to be a space interpreted as a symbol in language, given meaning by its patterns of recurrence in human use. Paraphrasing Wittgenstein, the meaning of a space is its use in the language, as understood in and through the activity of those who use it.

Their principle "Space is the opportunity; place is the understood reality" now can be seen in the same way that a pattern of sound waves can be a word, a curve of ink can form a letter, a move of the hand a subtle gesture. In each case, the former is a perceivable pattern in one or more physical phenomena, which has the potential to be used symbolically, while the latter is the symbol in language. We continually mix phenomena in our everyday communication, and spatial media are an essential part of that mix. While space has its unique characteristics that differentiate it from other media, it has no privileged position above or apart from them.

The differences between media are usually very obvious. The varying physical phenomena involved, and the approaches to relatedness and differentiation that are particular to each are to some extent what distinguish media and let us treat each medium as if it were an isolated individuated entity. On the other hand, we tend to overlook their similarities. Their common potential for use in language makes each medium of information representation potentially semiological, and therefore symbols in different media can become part of the same language. Hence we see 'appropriate behavioural framing' as the understanding of language, as semiological activity with culturally developed social and structural norms.

This standpoint, interweaving phenomena and media such as space, data and text, also offers an alternative view of the 'complex forms' of Harrison and Dourish. The first such form is the 'spaceless place,' such as Usenet news groups. Spaceless places are navigated and used by means of relationships that are non-spatial but that nevertheless support "the tension between connectedness and distinction that leads to placefulness." As they put it, different social norms make for different places without an underlying notion of space. The concept of spaceless places seems complex when media are conceived of as disjoint, but we see language as the underlying notion of both spatial and non-spatial media. Language unites them and makes them 'placeful' or understood.

In the second class of complex forms, 'hybrid spaces,' physical and virtual spaces merge or overlap. Actions in physical space may be represented in a media space via camera images, for example. While it may be difficult for the people involved to handle symbols such as gestures and postures that have been represented in a new medium, one should consider actions in technological and 'everyday' media as involving a common set of people, objects and symbols. Difficulty stems not so much from the fact that a combination of media is involved, but that the particular representations and interactions that combine those media, i.e. the overall design, can be a poor fit with the people and their activity. Parenthetically, we note that limited or transformed representation in technological media is a feature shared by 'everyday' media. The limited field of view of the eye and the compression of perspective, a city street's constraints on 
view and motion, and one's finite experience and current context—-these also limit and transform what one perceives and hence influence how one interprets the 'natural' world.

Rather than seeing hybrid spaces as a special case, we find it difficult to identify spaces that are not hybrid. Actions, words, gestures, concepts and goals that are not particular to one medium, workplace or information space are inevitably involved, and span such spaces because the same people are involved in them all. Media spaces, virtual worlds and all information representations would be useless if they did not overlap with and share references to the patterns of symbolic activity of verbal, written and gestural language, and hence with the activity in everyday physical space. As Harrison and Dourish themselves put it, "after all, a virtual world filled with virtual offices and virtual desks isn't populated by virtual people, but by real ones."

\section{City}

In the City project, we wish to work with something richer and more complex than a collection of isolated pieces of information and media. We also wish to move beyond the traditional systems of classification and categorisation that too often over-objectify information and activity. Driven by the theoretical standpoint outlined in the previous section, we are creating a growing and evolving body of individuals' paths or narratives through the people, places and things associated with the city of Glasgow and with cities in general. An initial collection of raw data includes images, textual descriptions, and references to locations in the city, to artefacts in museums and exhibitions, and to electronic resources such as web pages. We will allow this body of information to grow as people use it, making new associations between symbols and adding in new ones. These evolving inter-subjective patterns of association and use will thus complement the static or a priori categories, type systems and indices that partition artefacts, spaces and people, and we offer systems that afford information access based on both objective and subjective bases.

While the project will gradually extend the range of places and topics it handles, the initial focal point is Charles Rennie Mackintosh, the architect, designer and artist. A rich body of people, places and things related to Mackintosh exists here in Glasgow. Examples include his reconstructed house within the Hunterian Gallery and Museum, the exhibition room devoted to his life and work within the Lighthouse Centre, and of course the buildings he designed such as the Art School and the Lighthouse itself. The Hunterian and the Lighthouse have agreed to be partners and test sites in this project, and in other related projects within the six year, eight site and $£ 11,000,000$ consortium called 'Equator'. Equator (www.equator.ac.uk) is an 'interdisciplinary research collaboration' funded by the UK's Engineering \& Physical Sciences Research Council and involving computer scientists, sociologists, psychologists, artists and designers. University College London and the universities of Bristol, Glasgow, Nottingham and Southampton are taking part in the City project, with Glasgow as lead.

We do not consider the images and fragments of text as our fundamental or central data. We wish to avoid making yet another database full of sterile bits and pieces about dry exhibits, dead people and empty buildings. We represent the paths or narratives through such symbols, i.e. points of view and interpretation of how people have used them, possibly implicitly expressed, that in turn afford reinterpretation. Also, we are not focusing on more tightly structured or scripted activity characteristic of traditional work. To use Schmidt's metaphor, we are focusing on maps not scripts (Schmidt 1997) i.e. on resources for activity and interpretation. We mix the work of the author, curator or guide with the leisure of the tourist or visitor in the cultural city setting. We do not intend to make these paths just by ourselves, but to have a number of parties contribute their own interpretations of the 'raw' data and of existing paths. In the course of this creative process, they might wish to bring in additional images, references and so forth, and we will support this. We will begin with the official or 'high culture' views of Mackintosh and the city, with editors or authors including curators of several Mackintosh resources, workers within cultural information and institutions in Glasgow and a partner in a local design consultancy. Then we will open their paths or narratives to visitors and residents of the city from a number of communities and backgrounds, and activity will be recorded and added to the set of paths. 
We are now building a central information resource, accessible via a variety of media and devices, that stores the paths/narratives are stored in our system as well as the elements or fragments combined within them. While we have started with a static collection in our first prototype, this resource will later support additions, deletions, annotations and associations that are, ultimately, necessary to make the collection reflect ongoing use by a widening set of users. Here we draw on Southampton's experience in hypermedia systems, and work at Bristol and Glasgow with dynamic information services tailored to small handheld computers. Nottingham has developed a new and highly flexible middleware system, EQUIP, that offers a shared and distributed data modelling. This serves us well in integration work within the City project, and we expect that it will ease later collaboration and co-development with other Equator projects such as CityWide, a project centred in performance and involving Nottingham, Southampton and the theatre group Blast Theory.

Another piece of infrastructure that is used by one or more devices is a set of systems for location tracking. In the case of location in a virtual environment, each person's location is easily obtained from the VR system. In the case of someone using a hypermedia document system, 'informational location' in terms of the recently used documents and links can also be obtained relatively easily. More difficult is physical location in the city or in a room. We can use GPS, RF tags and simple radio 'pingers' to more roughly and intermittently track location in the city. We are initially working inside exhibition rooms, where we use more fine-grained and continuous ultrasound tracking from U. Bristol. We can track a person to within $20 \mathrm{~cm}$ at an update rate of roughly once per second. Note that a person can have a location or context that involves all three media. For example, he or she may be standing in a particular square in the city, reading a document describing its history, and using a virtual model of the city to fly around it and get a feel for the neighbouring streets and skyline. More generally, and reiterating a point from the earlier section, the project wishes to explore the interwoven and interdependent nature of such media, treating them as one holistic design medium, i.e. human activity, rather than as isolated parts. We do not treat any one aspect of location or context as ultimately dominant, but instead try to support one's shifting focus, use and combination of them.

We intend to afford access to paths via audio, wearable computers, tablets and VRs. In the first case, we would generate a stream of verbal and non-verbal audio, supplied to a phone or a computer that a person would carry. The audio would describe the artefacts close to the person, adjusting the information given based on his or her location and motion, and the choice of paths and expressions of interest in artefacts and information along the way. Steve Brewster at Glasgow has experience in using positional audio, that makes sound appear to emanate from a particular direction around the person's head. This can be used to guide the user and to aid discrimination amongst multiple sources of sound. In the second case, we refer to a wearable computer with relatively small graphical displays, audio interfaces and a variety of context sensors integrated into a 'CyberJacket', as developed by U. Bristol. These wearables are of particular applicability to places such as the Mackintosh Room, where we use available fine-grained tracking of location and direction. A tablet refers to a laptop-like computer with wireless network communications, and it may have greater computation and display facilities than a wearable. Again we can do some fine-grained tracking here, but we can show textual documents, 2D and 3D graphics and animation, and so forth. In VRs, we refer to more high-end graphics systems that convey sophisticated 3D graphical and audio display of virtual environments, including CAVEs (such as that of UCL and another Equator partner, the Glasgow Science Centre). Using such a system, a person would see a graphical model of the city with the paths overlaid, linking and interweaving the artefacts and spaces that make up the exhibition.

An important aspect of this variety of media and devices is that we expect people's activities in each medium to be perceptible in all media. This will mean that synchronous users, possibly using different media, are made aware of each other and can directly communicate with each other. An example would be that of a person walking along the physical Buchanan Street, with a wearable that is tracked. That person's activity would be represented inside a virtual model of the city. Someone in London, exploring that virtual city, would have his or her activity tracked too, as would someone in Iceland browsing the hypermedia documents about that street. The first person's wearable could show if the Londoner and Icelander were available for a chat, the Icelander's documents could have temporary annotations with similar information, and the Londoner would see graphical representations of the Glaswegian's and the Icelander's activity and 
approachability. Again, we see human activities that span and connect media as being at least as important as the characteristic design affordances that differentiate and distinguish particular media.

Our design approach also means that use of each medium would be recorded in the central information resource, and made available for interpretation by people using the system later. This asynchronous communication will be in the form of explicit annotations and additions, in graphically presented paths/narratives of earlier authors and visitors, and in contextually specific recommendations of people, places and things. In the latter case we would use Glasgow's Recer system (Chalmers et al. 1998) that makes recommendations by comparing each person's ongoing context with his or her past activity and/or the past activities of others. Past activity here is represented by a time-stamped log of locations, documents, artefacts and people that each person has interacted with in all of the media we can. Irrespective of the media involved in logging and display, our algorithms for searching, matching and recommending do not rely on distinctions between these original media. They rely on their patterns of co-occurrence in human activity and hence in common semiological use. It is this change in emphasis that lets recommendations bridge across media usually held as separate in information systems, and lets us work with this everyday mix of types rather than against it. For example, a person who has been looking at the original chairs and tables made by Mackintosh may get recommendations of other physical artefacts to look at, but also of digital documents and virtual locations that might be of interest. These recommendations may come from people who have never seen the physical furniture, but have explored related digital information.

At the time of writing this position paper, VR and handheld/wearable systems are now inter-operating so that a person using a PDA can see another person using the same room, and vice versa. We have not integrated a hypermedia interface to the same room yet, but will do within the next month. We will also integrate the recommender system within two months. We are currently collecting our first authored paths, and installing further equipment in the Lighthouse's Mackintosh room. Apart such technology development tasks, we have recently begun a study of the combination of traditional and new media, through a series of semi-structured interviews with curators and exhibition designers in a number of UK museums. We are also just about to start a sociological study of the activity and interaction of city visitors and residents. We continue to explore the contemporary philosophy of language, phenomenology, neurolinguistics and the evolution of language, and urban design theory. These studies and readings will feed into later system design work and in reflection on our first full prototype. We plan to be able to demonstrate this by September 2001, with synchronous and asynchronous awareness across all three media supported, paths interweaving symbols from all media and used as a resource for recommendations, and first experiences of non-Equator people with the system to report and discuss.

\section{References}

Chalmers, M., K. Rodden \& D. Brodbeck (1998): The Order of Things: Activity-Centred Information Access, In Proceedings of the $7^{\text {th }}$ World Wide Web Conference, Brisbane, Australia.

Chalmers, M. (2001): Awareness, Representation and Interpretation, to appear in J. CSCW.

Harrison, S. \& P. Dourish (1996): Re-Place-ing Space: The Roles of Place and Space in Collaborative Systems, In Proceedings of Conference on Computer Supported Cooperative Work, ACM Press.

Schmidt, K. (1997): Of Maps and Scripts: The Status of Formal Constructs in Cooperative Work, In Proceedings of Group 97, ACM Press, Phoenix, USA, pp. 138-147. 